\title{
Millimeter-wave Observations of Polyatomic Molecules in Diffuse Clouds
}

\author{
Harvey Liszt ${ }^{1}$, Robert Lucas ${ }^{2}$, and Jerome Pety ${ }^{2,3}$ \\ ${ }^{1}$ National Radio Astronomy Observatory, 520 Edgemont Road Charlottesville, VA 22903, USA \\ ${ }^{2}$ Institut de Radioastronomie Millimétrique, \\ 300 rue de la Piscine F38406 Saint Martin d'Hères, France \\ ${ }^{3}$ Observatoire de Paris, 61 av. de l'Observatoire, F75014 Paris, France
}

\begin{abstract}
Millimeter-wave (mm-wave) absorption profiles toward extragalactic sources consistently find just diffuse (occasionally perhaps translucent) neutral gas-low/moderate density and extinction - along even some very long, dark lines of sight. CO, often heavily fractionated and mimicking the appearance of dark gas in emission, occasionally absent in emission even when present in absorption, is not the dominant form of carbon in these regions (presumably it is $\mathrm{C}^{+}$) yet the abundances of many other molecules resemble those seen in TMC-1. Some species $\left(\mathrm{OH}, \mathrm{HCO}^{+}, \mathrm{C}_{2} \mathrm{H}\right.$ and $\left.\mathrm{C}_{3} \mathrm{H}_{2}\right)$ turn on with high abundances just when $\mathrm{H}_{2}$ does; others (HCN, HNC, CN) require slightly higher $N\left(\mathrm{H}_{2}\right)$ and yet others (CS and other sulfur-bearing species, $\mathrm{NH}_{3}$ and $\mathrm{H}_{2} \mathrm{CO}$ ) even higher $N\left(\mathrm{H}_{2}\right)$. The systematics and implications of these recent discoveries are discussed here.
\end{abstract}

Keywords. astrochemistry — ISM: clouds — ISM: molecules — ISM: structure

\section{Introduction}

Within a few years of the discovery of CO $J=1-0$ rotational emission from Orion (Wilson et al. 1970), a wealth of mm-wave observations quickly established the existence of complex polyatomic chemistries in dense interstellar clouds, largely driven by cosmicray ionization of $\mathrm{H}_{2}$ to form $\mathrm{H}_{3}{ }^{+}$(Herbst \& Klemperer 1973). The rest is, if not exactly history, at least the subject of this well-attended meeting on interstellar chemistry. Today, observations of molecular rotational emission are synonymous with the idea of "molecular gas" or "molecular clouds", and such emission traces the star-forming gas reservoirs both nearby and in the most distantly-observable systems in the Universe.

However, the existence of molecules in the ISM was first noted nearly 70 years ago in optical spectra of regions which are not at all heavily extinguished or very dense: accumulating gradually over this long interval, the list of molecules observed in such "diffuse gas" or "diffuse clouds" is both fairly extensive and in some regards very difficult to understand. Recently, the pace of observational discovery has quickened considerably owing to the application of mm-wave absorption techniques to difuse cloud studies, which is the subject of this contribution.

\section{Precedents for RF Observations of Molecules in Diffuse Clouds}

It was the observation of $\lambda 18 \mathrm{~cm} \mathrm{OH}$ emission from dark clouds (Heiles 1969) which as much as anything else first convincingly showed the prevalence of $\mathrm{H}_{2}$ in such regions, where the $\mathrm{H}$-nuclei (directly observed only in $\mathrm{HI}$ ) seemed otherwise untraceable. Thereafter, surveys of $\mathrm{OH}$ emission and absorption in directions marked by known $\mathrm{H} \mathrm{I}$ absorption certainly and often knowingly sampled molecular gas in diffuse clouds (Dickey et al. 1981), but provided little opportunity for follow-up. When CO and $\mathrm{CH}$ emission 

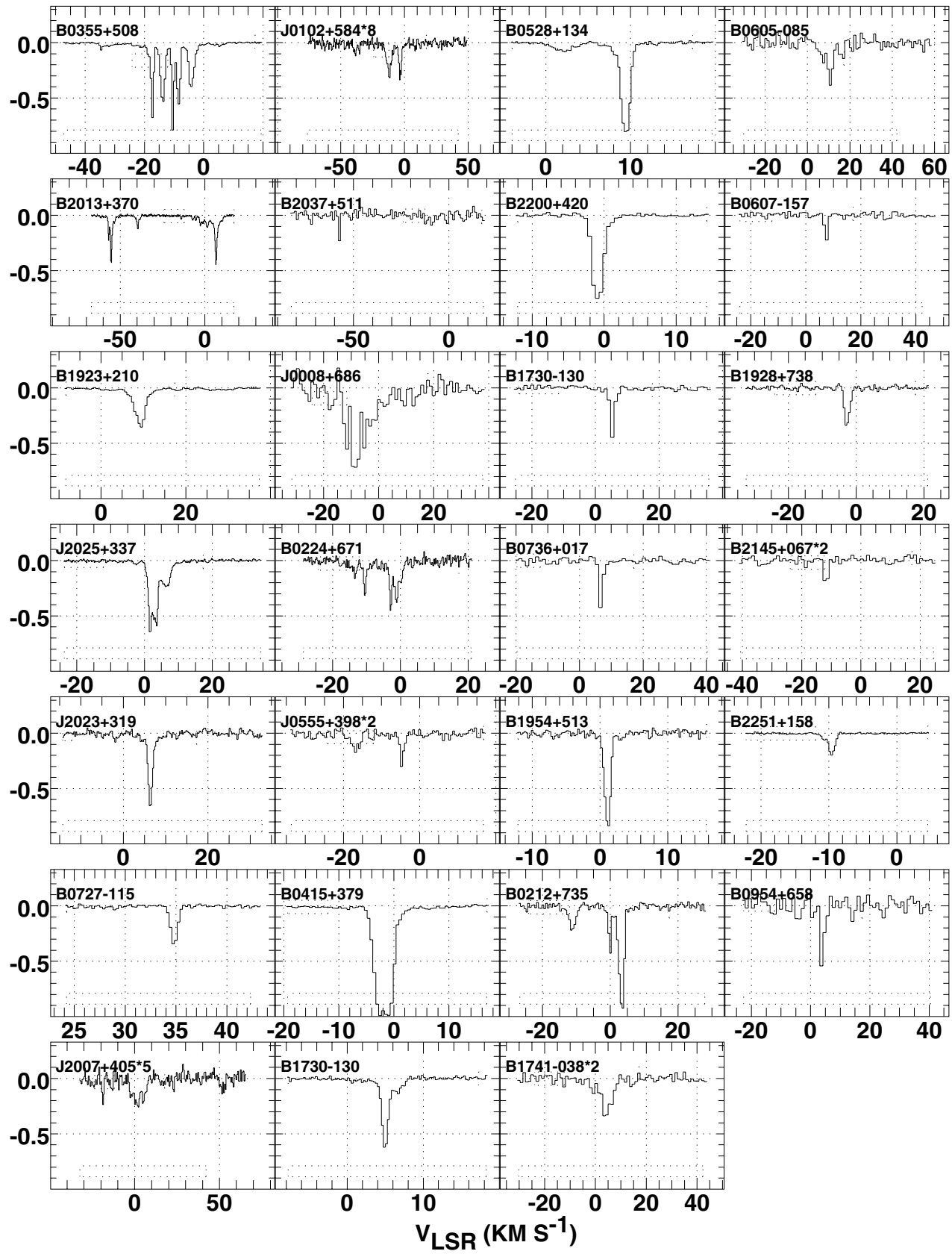

Figure 1. Rogue's gallery of all $\mathrm{HCO}^{+}$profiles.

were observed in diffuse clouds with high resolution, as toward $\zeta$ Oph (Liszt 1979), the observed line profiles were narrower than optical spectroscopists had in general been willing to consider, but such studies also cannot be said to have had much influence because the abundaces of $\mathrm{CO}$ and $\mathrm{CH}$ were more straightforwardly determined in optical/UV absorption spectra. 
With hindsight it becomes obvious that radio-frequency observations of molecules in diffuse gas were often not recognized as such when first performed - for instance, in $\mathrm{H}_{2} \mathrm{CO}$ absorption surveys over the galactic plane (see the remarks in Liszt \& Lucas 1995) and in spectra of $\mathrm{NH}_{3}$ and $\mathrm{H}_{2} \mathrm{CO}$ in the work of Nash (1990)-largely owing to disbelief that such species would be detectable there (see Federman \& Allen 1991). But by the end of the 1980's, with PAHs becoming an increasing topic of discussion, Cox et al. (1988) certainly and tellingly stated the matter quite clearly in showing the ubiquity of the hydrocarbon $\mathrm{C}_{3} \mathrm{H}_{2}$ in the diffuse ISM.

Marscher et al. (1991) subsequently showed that sensitive mm-wave absorption profiles could be taken with small arrays $(3 \times 10 \mathrm{~m})$. Specifically, using OVRO, they produced a profile of ${ }^{12} \mathrm{CO} J=1-0$ absorption toward BL Lac, an (approximately) $1 \mathrm{Jy} \mathrm{mm}$ continuum source which was known to suffer an optical extinction about equal to that of $\zeta$ Oph (0.32 mag) and which had long been used as a target for $\mathrm{H}$ I and $\mathrm{OH}$ absorption studies. The line was, unfortunately, quite optically thick, complicating their efforts to infer the physical structure of the intervening medium, but it sufficed to demonstrate the practicality of taking such spectra.

We subsequently were in the process of observing ${ }^{13} \mathrm{CO}$ absorption against BL Lac and other compact extragalactic mm-wave sources toward which $\mathrm{CO}$ had been seen in emission surveys (Liszt \& Wilson 1993; Liszt 1994), when two fortuitous events intervened: we became aware of a serendipitous detection of $\mathrm{HCO}^{+}$absorption toward $\mathrm{BL}$ Lac during an unrelated instrumental phase calibration for another project, and BL Lac flared (actually not an uncommon event among blazars). We asked for time to observe $\mathrm{HCO}^{+}$and other species, and found them surprisingly handily toward BL Lac and elsewhere (Lucas \& Liszt 1993, 1994). What is discussed here follows from these two happy coincidences now back a baker's dozen of years.

\section{3. $\mathrm{HCO}^{+}$and Implications for Diffuse Cloud Chemistry}

We surveyed $\mathrm{HCO}^{+}$absorption toward an approximately flux-limited sample of a few dozen strong background sources (this is complicated when all the sources are variable) and reported the results in Lucas \& Liszt (1996). A slightly updated version of those results is shown in the first two figures. Figure 1 shows the actual absorption profiles we have accumulated, in order (left to right and top to bottom) of increasing galactic latitude $(|b|)$. The profiles are summarized in two ways in Figure 2, where the profile integrals, normalized to column density assuming excitation at the temperature of the cosmic background, are plotted twice. At left, we illustrate our old result that $\mathrm{HCO}^{+}$ absorption was found in essentially all directions at galactic latitudes below about $15^{\circ}$, but much less commonly above; although $\mathrm{HCO}^{+}$absorption is somewhat more frequent than $\mathrm{CO}$ emission, the same effect had been seen in CO surveys and it is attributable to the local galactic geometry. Basically, because of the "local bubble" we look out as from the bed of a wide but shallow crater; to look out in unobscured fashion it is necessary also to look up (or down) some $12^{\circ}-15^{\circ}$. The local neutral gas is dominated not by a plane parallel vertical stratification but by the excavatory efforts of the bubble.

At right in Figure 2 the same $\mathrm{HCO}^{+}$integrals are plotted against the reddening taken from the work of Schlegel et al. (1998). From this, four particular physical inferences can be drawn: (1) The absorption becomes detectable rather abruptly at $E_{\mathrm{B}-\mathrm{V}}>0.08$ mag, which is the famous turn-on of high $\mathrm{H}_{2}$-fractions in the diffuse ISM according to Bohlin et al. (1978); see also Lucas \& Liszt (2000b). (2) At higher reddening, $N\left(\mathrm{HCO}^{+}\right)$ increases linearly with $E_{\mathrm{B}-\mathrm{V}}$ suggesting that $\mathrm{HCO}^{+}$turns on, at full-strength, about when $\mathrm{H}_{2}$ does. (3) The $\mathrm{HCO}^{+}$abundance is very large, nearly as high as in TMC-1, 

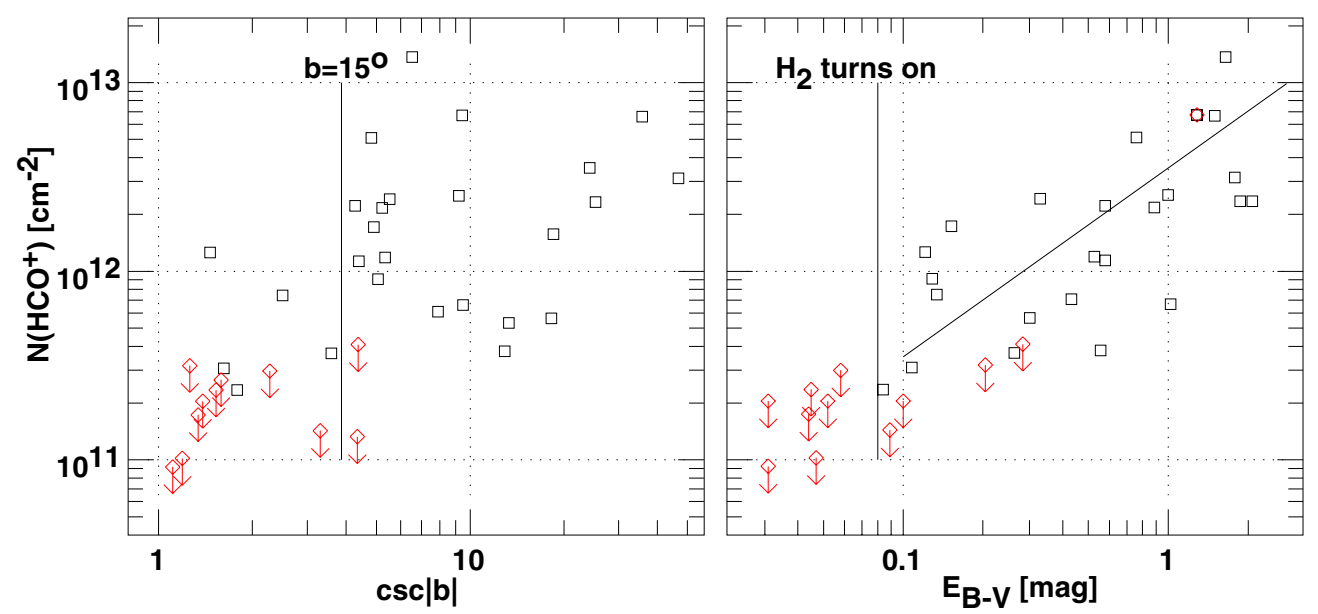

Figure 2. Total $\mathrm{HCO}^{+}$column density vs. $\csc (|b|)$ and $E_{\mathrm{B}-\mathrm{V}}$.

and (4) much smaller abundances are predicted by conventional gas-phase chemistry of diffuse clouds; for (3) and (4) see just below.

Using the usual gas-reddening ratio, whereby $E_{\mathrm{B}-\mathrm{V}}=1$ mag corresponds to a column density $N(\mathrm{H})=5.8 \times 10^{21} \mathrm{~cm}^{-2}$ (Bohlin et al. 1978), we have that $N\left(\mathrm{HCO}^{+}\right) / E_{\mathrm{B}-\mathrm{V}}$ $\approx 3 \times 10^{12} \mathrm{mag}^{-1}$ or $N\left(\mathrm{HCO}^{+}\right) / N(\mathrm{H})=5 \times 10^{-10}$. Then, if the typical fraction of $\mathrm{H}$-nuclei in $\mathrm{H}_{2}$ is 0.2 in the diffuse ISM, as per the Copernicus observations, it follows that $X\left(\mathrm{HCO}^{+}\right)=N\left(\mathrm{HCO}^{+}\right) / N\left(\mathrm{H}_{2}\right)=5 \times 10^{-9}$; the usual value quoted for TMC-1 is $X\left(\mathrm{HCO}^{+}\right)=10^{-8}$ (Ohishi et al. 1992). In fact in our work we typically use smaller values, $X\left(\mathrm{HCO}^{+}\right)=2-3 \times 10^{-9}$, but these also are far too large to be easily understood.

In diffuse gas, the dominant form of gas-phase carbon is $\mathrm{C}^{+}$, not $\mathrm{CO}$, somewhat turning dense-gas CO chemistry on its head (Glassgold \& Langer 1976; Black \& Dalgarno 1977). That is, conventional gas-phase chemistry forms $\mathrm{CO}$ by first producing $\mathrm{CO}^{+}$via $\mathrm{C}^{+}+$ $\mathrm{OH} \rightarrow \mathrm{CO}^{+}+\mathrm{H}$, after which $\mathrm{CO}$ forms either by $\mathrm{CO}^{+}+\mathrm{H} \rightarrow \mathrm{CO}+\mathrm{H}$, or by $\mathrm{CO}^{+}+$ $\mathrm{H}_{2} \rightarrow \mathrm{HCO}^{+}+\mathrm{H}, \mathrm{HCO}^{+}+\mathrm{e} \rightarrow \mathrm{CO}+\mathrm{H}$. All these reactions are relatively fast, and radiative recombination $\mathrm{CO}^{+}+\mathrm{e} \rightarrow \mathrm{C}+\mathrm{O}$ is unimportant with the $\mathrm{e} / \mathrm{H}$ fractions expected for diffuse gas (Liszt 2003). So $\mathrm{CO}$ is the dominant product of the $\mathrm{C}^{+}-\mathrm{OH}$ interaction no matter the $\mathrm{H}_{2}$-fraction, but, if the region is substantially molecular, most $\mathrm{CO}^{+}$will proceed to $\mathrm{HCO}^{+}$. Then, because $\mathrm{n}(\mathrm{e}) \approx \mathrm{n}\left(\mathrm{C}^{+}\right)$(ibid.), it follows pretty directly that the $\mathrm{OH} / \mathrm{HCO}^{+}$ratio will be largely independent of the density and $\mathrm{H}_{2}$-fraction etc. and somewhat dependent on temperature owing to the functional dependence of the $\mathrm{HCO}^{+}$recombination rate coefficient. With values $X(\mathrm{OH}) / X\left(\mathrm{HCO}^{+}\right) \approx 1000$ for typical conditions, it follows that $X\left(\mathrm{HCO}^{+}\right)=10^{-10}$ if $X(\mathrm{OH})=10^{-7}$ as observed along a few sightlines (Felenbok \& Roueff 1996; Liszt \& Lucas 2002).

So the discrepancy between observation and theory is quite substantial, if not as extreme as for $\mathrm{CH}^{+}$. The column densities of $\mathrm{C}^{+}, \mathrm{OH}$, and $\mathrm{CO}$ are all observable in optical/UV absorption, so the profound failure of this scheme to explain either $X\left(\mathrm{HCO}^{+}\right)$or $X(\mathrm{CO})$ would ordinarily lead to its dismissal. However, a fixed ratio $N\left(\mathrm{HCO}^{+}\right) / N(\mathrm{OH})$ is actually observed, and no generally acepted alternatives for explaining either this ratio or $X\left(\mathrm{HCO}^{+}\right)$and $X(\mathrm{CO})$ have appeared. The high abundances of $\mathrm{H}_{3}{ }^{+}$discussed by McCall at this meeting do not by themselves account for the creation of $\mathrm{HCO}^{+}$from $\mathrm{CO}$, by large factors. 


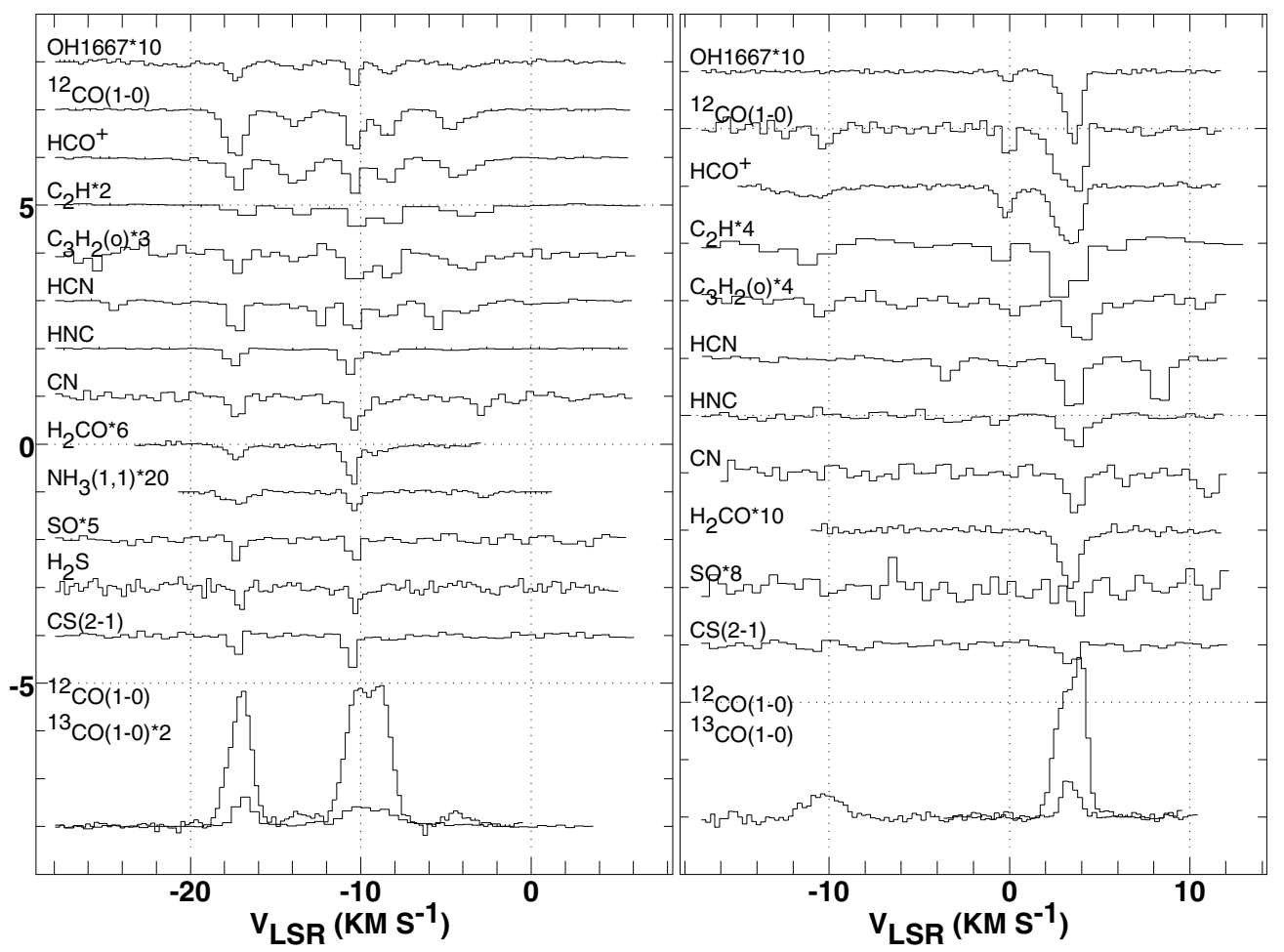

Figure 3. Left: Spectra toward B0355 +508 at $b=-1.6^{\circ}$. Right: B0212 +735 at $b=12.0^{\circ}$.

Although not understood, the observation of a high abundance of $\mathrm{HCO}^{+}$is by itself sufficient to eliminate yet another failure of chemical models, to explain the abundance of CO itself (Van Dishoeck \& Black 1988). If an abundance $X\left(\mathrm{HCO}^{+}\right)=2-3 \times 10^{-9}$ can be maintained, the usual radiative recombination to form $\mathrm{CO}$ is sufficient to account for the observed $X(\mathrm{CO})$ when the formation of $\mathrm{H}_{2}$ and $\mathrm{CO}$ is calculated self-consistently, including their self and mutual shielding (see Liszt \& Lucas 2000). But the solution of the $\mathrm{CO}$ problem is actually somewhat more robust than even this discussion indicated, because $\mathrm{CO}^{+}+\mathrm{H} \rightarrow \mathrm{CO}+\mathrm{H}^{+}$may form $\mathrm{CO}$ without first forming $\mathrm{HCO}^{+}$. Molecular chemistry in only partially-molecular regions is really more different than at first might meet the eye.

\section{Chemical Complexity and Systematics in Diffuse Clouds}

Figure 3 shows an exhaustive summary of our molecular profiles taken toward two sources with rich spectra. The most commonly occurring species- $\mathrm{OH}, \mathrm{HCO}^{+}(\mathrm{Liszt} \&$ Lucas 1996; Lucas \& Liszt 1996), CO (Liszt \& Lucas 1998), $\mathrm{C}_{2} \mathrm{H}$ and $\mathrm{C}_{3} \mathrm{H}_{2}$ (Lucas \& Liszt 2000a) - appear at top, followed by the CN-family (Liszt \& Lucas 2001), which is slightly more common than those further below, namely, $\mathrm{H}_{2} \mathrm{CO}, \mathrm{NH}_{3}$ (see Lucas \& Liszt 1995; Nash 1990; and a forthcoming paper of ours) and the sulfur-bearing species (Lucas \& Liszt 2002).

The long line of sight toward B0355 at $b=-1.6^{\circ}$ is itself dark $\left(E_{\mathrm{B}-\mathrm{V}}=1.5 \mathrm{mag}\right)$, but the features seen along it are most likely diffuse; there are about a half-dozen of these with very nearly the same $N\left(\mathrm{HCO}^{+}\right) \approx 1-1.5 \times 10^{12} \mathrm{~cm}^{-2}$ and $N(\mathrm{OH}) \approx 3-5 \times 10^{13} \mathrm{~cm}^{-2}$, just as toward $\zeta$ Oph and other classic optical diffuse-cloud lines of sight (see Fig. 4). 
The absorption features generally appear in CO emission (bottom) albeit rather weakly in some. Of the three absorption features which emit more strongly in ${ }^{12} \mathrm{CO}$, two are chemically much richer than the other.

At right, toward $\mathrm{B} 0212$ at $b=12^{\circ}\left(E_{\mathrm{B}-\mathrm{V}}=0.76 \mathrm{mag}\right)$ the strongest absorption component has higher column densities than any feature seen toward B0355, while the absorption feature at 0-velocity lacks a $\mathrm{CO}$ emission counterpart at quite low levels. This is an example of the fact that $\mathrm{CO}$ and some polyatomics form at much lower pressure than is needed to excite even $\mathrm{CO}$ to detectable levels in emission. A search for $\mathrm{HCO}^{+}$emission revealed only a single very weak $(0.02 \mathrm{~K})$ line toward each source (Lucas \& Liszt 1996).

$\mathrm{HCO}^{+}$emission at such low levels is consistent with rather low density when due to electron excitation in a gas where the carbon is largely in the form of $\mathrm{C}^{+}$. That is, for a typical thermal pressure of $p / \mathrm{k}=2000-3000 \mathrm{~cm}^{-3} \mathrm{~K}$, at temperatures $30-70 \mathrm{~K}, n(\mathrm{H}) \approx$ 30-100, with $n(\mathrm{e}) / n(\mathrm{H}) \approx 2 \times 10^{-4}$ (Liszt \& Lucas 1994; Lucas \& Liszt 1996). Consistent with this, ${ }^{13} \mathrm{CO}$ is often observed to be heavily fractionated into $\mathrm{CO}$ in our work, yielding relative abundance ratios $N\left({ }^{12} \mathrm{CO}\right) / N\left({ }^{13} \mathrm{CO}\right)$ as small as $12-15$ (Liszt \& Lucas 1998) in gas where the isotope ratio is actually 60-65 (Lucas \& Liszt 1998). In one case the fractionation is so strong that $\mathrm{HCN}$ appears to be starved for ${ }^{13} \mathrm{C}$ (ibid.) Observations of CO toward hot stars seem to show the opposite effect of selective photodissociation of ${ }^{13} \mathrm{CO}$ (see the contribution by Snow in this volume).

\section{Implications for Interpretation of CO Emission}

For weak excitation (say, a few $\mathrm{K}$ above the CMB) a feature with $N(\mathrm{CO}) \approx 10^{15} \mathrm{~cm}^{-2}$ will have an optical depth of unity in the $J=1-0$ line over a span of $1 \mathrm{~km} \mathrm{~s}^{-1}$ (Liszt \& Lucas 1998); the optical depth of the $J=1-0$ line of CO toward $\zeta$ Oph should be about 2 , according to optical spectroscopy. The point is that even diffuse clouds with relatively small amounts of carbon in the form of $\mathrm{CO}$ may have quite optically thick lines, and, depending on internal conditions, they may emit either fairly strongly or not at all. Examples of both sorts of behaviour are plentiful in our data. In both cases shown in Figure 3, the CO column densities of even the stronger features are small compared to the amount of free gas-phase carbon in a gas column having $A_{\mathrm{V}}=1 \mathrm{mag}$, i.e. $N(\mathrm{CO})$ $\approx 10^{16} \mathrm{~cm}^{-2}$ even toward B0212 (Liszt \& Lucas 1998). Yet, they emit at levels $(3 \mathrm{~K})$ typically associated with dark gas. What is more, the ${ }^{13} \mathrm{CO} /{ }^{12} \mathrm{CO}$ ratios seen in emission are often "fake", resulting from fractionation but giving the impression of arising from very optically thick $\mathrm{CO}$.

\section{Chemical Systematics}

Figure 4 shows that the chemistry is well-organized, however mystifying. At upper left we have copied a diagram from our initial survey work of $\mathrm{OH}$ and $\mathrm{HCO}^{+}$ (Liszt \& Lucas 1996; Lucas \& Liszt 1996) illustrating the very tight empirical relationship between the integrated optical depths of $\mathrm{OH}$ and $\mathrm{HCO}^{+}$. To quote $N(\mathrm{OH})$ it is necessary to ascertain the excitation temperature, which we did based on a comparison of $\mathrm{OH}$ emission and absorption results; the same small excitation temperatures are seen optically (Felenbok \& Roueff 1996) and in fact have been a puzzle for nearly 25 years. The $\mathrm{OH}$ should be thermalized in collisions with electrons in diffuse gas as noted by Dickey et al. (1981). Parenthetically, we note that the same discrepancy appears in $\mathrm{H}_{2} \mathrm{CO}$, which appears in anomalous absorption in some directions in our work (Liszt \& Lucas 1995): the $\lambda 6 \mathrm{~cm}$ transition, too, should be thermalized. 

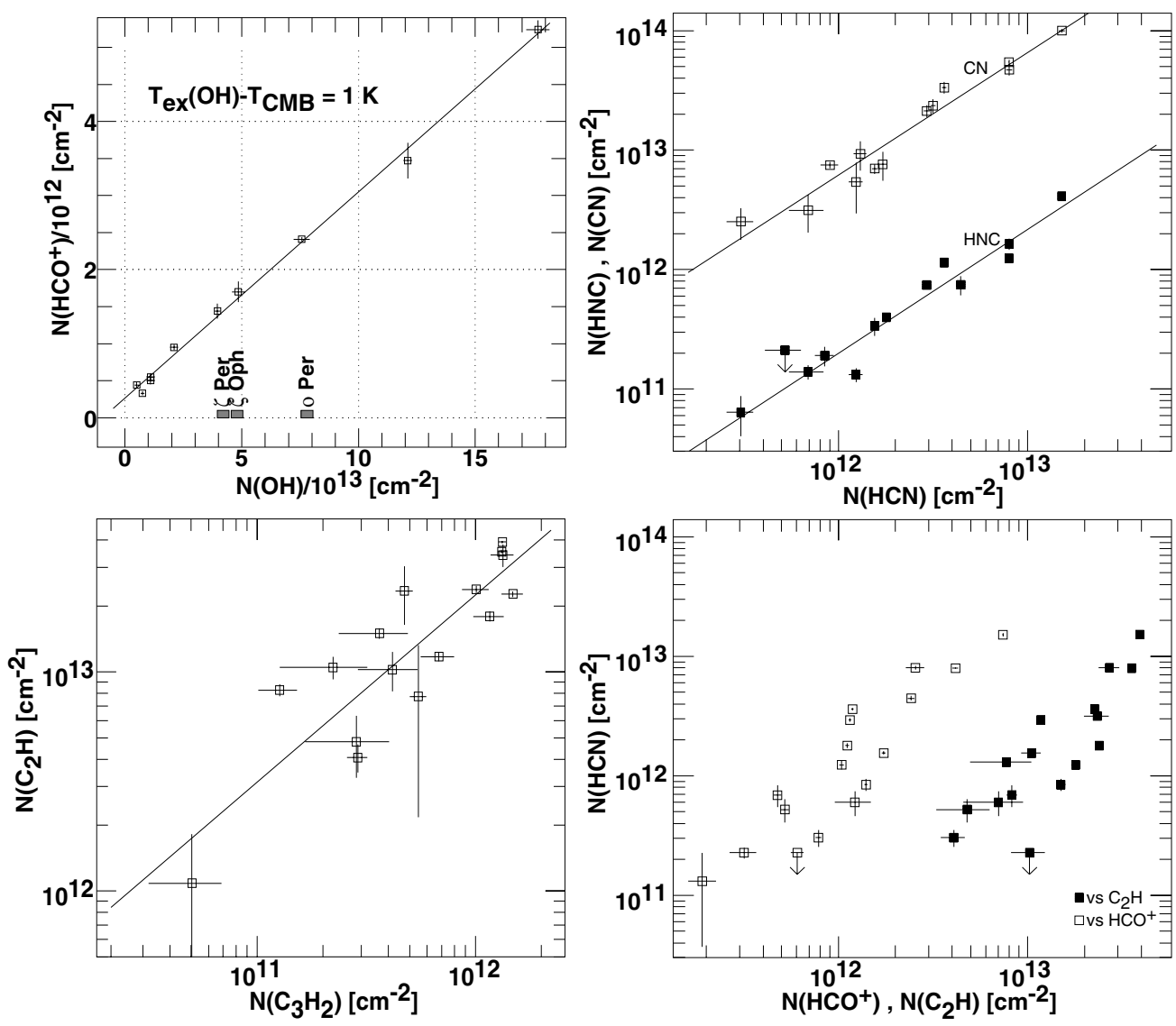

Figure 4. Relationships between chemical species and families.

In any case, the relationship expected between $\mathrm{OH}$ and $\mathrm{HCO}^{+}$is manifest, although with $N(\mathrm{OH}) / N\left(\mathrm{HCO}^{+}\right) \approx 30-50$, rather than 1000 or more. Also noted in that diagram are the $\mathrm{OH}$ column densities along three of the four directions where it is seen optically; clearly our clouds span the same regime. Later versions of this diagram had a bit more scatter. The $\mathrm{OH} / \mathrm{HCO}^{+}$abundance ratio toward TMC-1, far off to the right at $N(\mathrm{OH})=$ $2.5 \times 10^{15} \mathrm{~cm}^{-2}$ (Ohishi et al. 1992), is only $50 \%$ higher than given by the extrapolation of the fit shown here.

Other panels in Figure 4 show that there are similarly strong linear relationships in other chemical families as well $\left(\mathrm{CN}, \mathrm{HCN}, \mathrm{HNC} ; \mathrm{C}_{2} \mathrm{H}\right.$ and $\left.\mathrm{C}_{3} \mathrm{H}_{2}\right)$ and, generally strong non-linearities otherwise. No species beside $\mathrm{OH}$ is linear with $\mathrm{HCO}^{+}$, and none beside $\mathrm{C}_{3} \mathrm{H}_{2}$ with $\mathrm{C}_{2} \mathrm{H}$. CS is approximately linear with $\mathrm{HCN}$ and $\mathrm{NH}_{3}$ is linear with $\mathrm{H}_{2} \mathrm{CO}$. The steep slope of HCN's variation with $\mathrm{HCO}^{+}$around $N\left(\mathrm{HCO}^{+}\right)=1-3 \times 10^{12} \mathrm{~cm}^{-2}$ in Figure 4 can be directly related to the turn-on of $\mathrm{CN}$ with $\mathrm{H}_{2}$ in optical data compiled by Federman (e.g., Federman et al. 1994). The large HCN/HNC ratios seen in our work are typical of warm gas, i.e. not especially like TMC-1 and other dark clouds, but, except in this regard, the abundance ratios are all too often astoundingly like those quoted for TMC-1. 


\section{Conclusions}

We began these studies by trying to detect ${ }^{13} \mathrm{CO}$ absorption in classic "molecular" gas, for the purpose of understanding CO line formation. Instead, we stumbled upon the existence of an unexpectedly rich chemistry in diffuse clouds, repeating the experience of optical observers 60 years earlier. Although our directions were at first chosen blindly (in a flux-limited sample) and although many of our sightlines at low latitude are long and very dark, we have been unable to find any dense or dark gas. But perhaps we found the next best thing. The abundances we derived for $\mathrm{HCO}^{+}, \mathrm{C}_{2} \mathrm{H}$ and $\mathrm{C}_{3} \mathrm{H}_{2}$ generally, or (for example) for $\mathrm{HCN}, \mathrm{CS}, \mathrm{NH}_{3}$ etc. in the richer regions, are surprisingly (and mystifyingly) like those seen toward TMC-1 (the $\mathrm{HNC} / \mathrm{HCN}$ and $\mathrm{HOC}^{+} / \mathrm{HCO}^{+}$ratios are typical of warm gas). And although the diffuse gas is largely silent in emission, even in some cases in $\mathrm{CO}$, its $\mathrm{CO}$ emission may also be surprisingly strong and, due to the peculiar chemistry of carbon isotope fractionation, also somewhat misleadingly dark-cloud like.

Although clearly following some well-worn paths, we sincerely hope that the chemical problems elucidated in our work are not as persistent as, say, that for $\mathrm{CH}^{+}$. In summarizing the conference, Alex Dalgarno noted that the appearance of a dark-cloud-like set of chemical abundances in diffuse gas might be a clue that the chemistry was okay (his word) and the solution is to be found in gas dynamics (shock-dynamical models have been unsuccessful for $\mathrm{CH}^{+}$, of course). Or perhaps he said demonics (as in Maxwell's); we were sitting somewhat off to the side. There are a number of strong hints that the diffuse neutral ISM is not the simple undifferentiated woofly thing of idealized models. Linewidths are somewhat supersonic; some studies show very small scale structure (10$100 \mathrm{AU}$ ) in optical and RF absorption; there is a ubiquitous small fraction of material at very high pressure in $\mathrm{C}$ I excitation studies; the excitation of most $\mathrm{OH}$ and some $\mathrm{HCO}^{+}$ seems to be oblivious to the high electron fractions in diffuse gas.

Diffuse clouds, usually noted for and defined by their seeming transparency, nonetheless manage to have a rich interior life to which we are not entirely privy, and it is these presently hidden aspects, not our idealizations, that are most important to understanding what is happening in them.

\section{Acknowledgements}

The National Radio Astronomy Observatory is operated by AUI, Inc. under a cooperative agreement with the US National Science Foundation. IRAM is operated by CNRS (France), the MPG (Germany) and the IGN (Spain). We owe the staff at IRAM (Grenoble) and the Plateau de Bure our thanks for their assistance in taking the data.

\section{References}

Black, J. H. \& Dalgarno, A. 1977, Ap. J., Suppl. 34, 405

Bohlin, R. C., Savage, B. D., \& Drake, J. F. 1978, Ap. J. 224, 132

Cox, P., Güsten, R., \& Henkel, C. 1988, A\&ऽA 206, 108

Dickey, J. M., Crovisier, J., \& Kazes, I. 1981, A\&A 98, 271

Federman, S. R. \& Allen, M. 1991, Ap. J. 375, 157

Federman, S. R., Strom, C. J., Lambert, D. L., Cardelli, J. A., Smith, V. V., \& Joseph, C. L. 1994, Ap. J. 424, 772

Felenbok, P. \& Roueff, E. 1996, Ap. J. 465, L57

Glassgold, A. E. \& Langer, W. D. 1976, Ap. J. 206, 85

Heiles, C. 1969, Ap. J. 157, 123

Herbst, E. \& Klemperer, W. 1973, Ap. J. 185, 505

Liszt, H. 1979, Ap. J. 233, L147

Liszt, H. 1994, Ap. J. 429, 638 
Liszt, H. 2003, A\&̈A 398, 621

Liszt, H. \& Lucas, R. 1994, Ap. J. 431, L131

Liszt, H. \& Lucas, R. 1995, A\&A 299, 847

Liszt, H. \& Lucas, R. 1996, A\&A 314, 917

Liszt, H. \& Lucas, R. 1998, A\&A 339, 561

Liszt, H. \& Lucas, R. 2000, A\&A 355, 333

Liszt, H. \& Lucas, R. 2001, $A \mathscr{G} A 370,576$

Liszt, H. \& Lucas, R. 2002, A\&A 391, 693

Liszt, H. \& Wilson, R. W. 1993, Ap. J. 403, 663

Lucas, R. \& Liszt, H. 1993, A\&A 276, L33

Lucas, R. \& Liszt, H. 1994, A\&A 282, L5

Lucas, R. \& Liszt, H. 1996, A\&A 307, 237

Lucas, R. \& Liszt, H. 1998, A\& A 337, 246

Lucas, R. \& Liszt, H. 2000a, A\&BA 358, 1069

Lucas, R. \& Liszt, H. 2000b, A\&̈A 355, 327

Lucas, R. \& Liszt, H. 2002, A\&GA 384, 1054

Marscher, A. P., Bania, T. M., \& Wang, Z. 1991, Ap. J. 371, L77

Nash, A. G. 1990, Ap. J., Suppl. 72, 303

Ohishi, M., Irvine, W., \& Kaifu, N. 1992, in Astrochemistry of cosmic phenomena: proceedings of the 150th Symposium of the International Astronomical Union, held at Campos do Jordao, Sao Paulo, Brazil, August 5-9, 1991. Dordrecht: Kluwer, ed. P. D. Singh, 171-172

Schlegel, D. J., Finkbeiner, D. P., \& Davis, M. 1998, Ap. J. 500, 525

Van Dishoeck, E. F. \& Black, J. H. 1988, Ap. J. 334, 771

Wilson, R. W., Jefferts, K. B., \& Penzias, A. A. 1970, Ap. J. 161, L43 


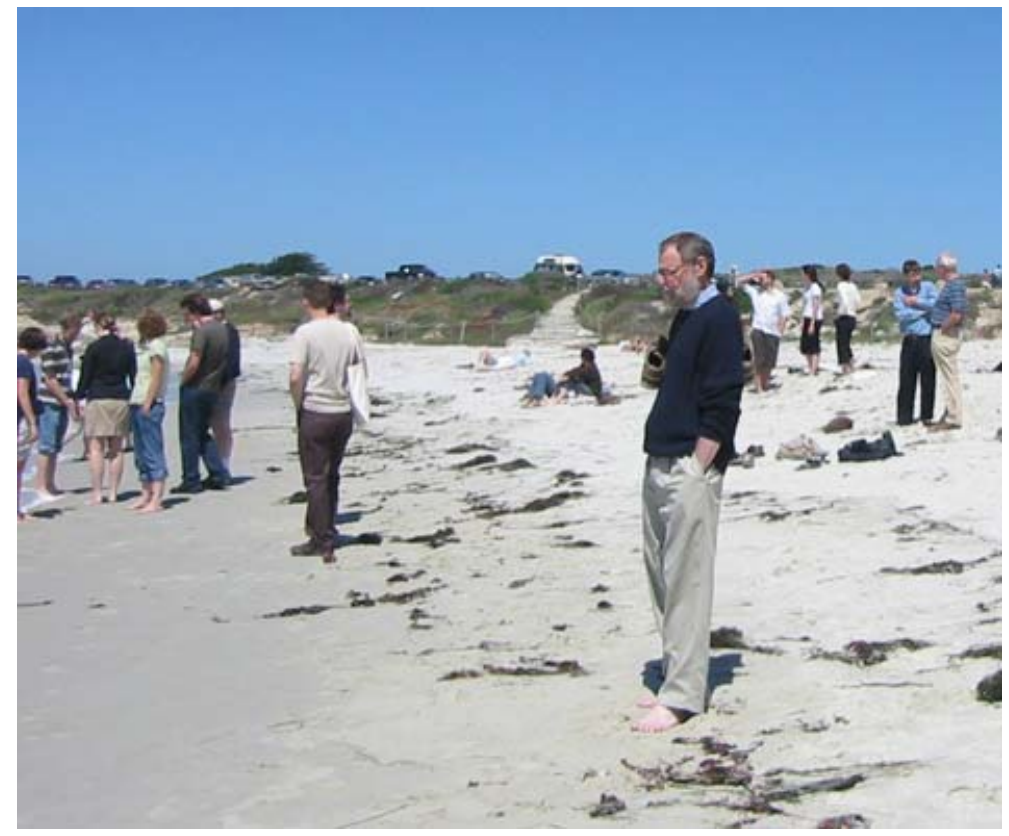

Photo: E. van Dishoeck 